\title{
UCRL 7192
}

\section{University of California}

MASI

\section{Ernest 0 . Lawrence Radiation Laboratory}

SCATTERING OF 14-MeV NEUTRONS

FROM NITROGEN AND OXYGEN

Livermore, California 


\section{DISCLAIMER}

This report was prepared as an account of work sponsored by an agency of the United States Government. Neither the United States Government nor any agency Thereof, nor any of their employees, makes any warranty, express or implied, or assumes any legal liability or responsibility for the accuracy, completeness, or usefulness of any information, apparatus, product, or process disclosed, or represents that its use would not infringe privately owned rights. Reference herein to any specific commercial product, process, or service by trade name, trademark, manufacturer, or otherwise does not necessarily constitute or imply its endorsement, recommendation, or favoring by the United States Government or any agency thereof. The views and opinions of authors expressed herein do not necessarily state or reflect those of the United States Government or any agency thereof. 


\section{DISCLAIMER}

Portions of this document may be illegible in electronic image products. Images are produced from the best available original document. 


\section{UNIVERSITY OF CALIFORNIA \\ Lawrence Radiation Laboratory}

Livermore, California

Contract No. W-7405-eng- 48

SCATTERING OF 14-MeV NEUTRONS FROM NITROGEN AND OXYGEN

R. W. Bauer

J. D. Anderson

L. J. Christensen

February 27, 1963

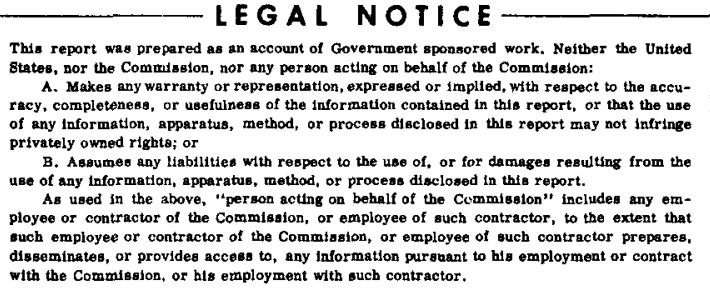




\section{Scattering of $14-\mathrm{MeV}$ neutrons from nitrogen and oxygen \\ R. W. Bauer, J. D. Anderson and L. J. Christensen \\ Lawrence Radiation Laboratory, University of California \\ Livermore, California \\ February 27, 1963}

Abstract: The differential cross sections for elastic scattering of $14-\mathrm{MeV}$ neutrons from nitrogen and oxygen have been measured in the angular range from 17 to $140^{\circ}$, using liquid targets and annular ring geometry. Inelastic scattering to the 2.31 - and $3.95-\mathrm{MeV}$, levels in $\mathrm{N}^{14}$ and to the levels near 6 and $7 \mathrm{MeV}$ in $\mathrm{O}^{16}$ has been investigated over a more restricted angular range. The $2.31-\mathrm{MeV}$ level in $\mathrm{N}^{14}$ is not excited appreciably, in disagreement with previously reported results. For both elements, the elastic scattering cross sections (determined to an accuracy of $10 \%$ ) were found to be appreciably higher than the optical model predictions by Bjorklund and Fernbach for scattering angles larger than $70^{\circ}$. The inelastic cross sections measured are, within experimental accuracy, the same as the corresponding ( $\left.p, p^{\prime}\right)$ cross sections.

\section{Introduction}

During the past few years differential elastic scattering of $14-\mathrm{MeV}$ neutrons by light nuclei has been studied experimentally by several investigators $^{1-4}$ ). In this region of the periodic table definite deviations of the experimental data from the theoretical optical model predictions by Bjorklund and Fernbach ${ }^{5}$ ) have been reported, in particular for large scattering angles, while the same model has produced excellent agreement for elastic scattering by nuclei above aluminium ${ }^{6}$ ). Although it is possible to produce a 
theoretical angular distribution to fit each set of experimental data by a proper adjustment of the optical model parameter, as has been demonstrated by Nodvik et al. 7 ) for the case of carbon, further experimental investigations of elastic scattering by light nuclei appear to be extremely helpful for achieving a systematic compilation of the shape and the parameters of the complex potential describing such a model over a wider range of nuclei.

The investigation reported in this paper was undertaken to measure the differential elastic cross sections of $14-\mathrm{MeV}$ neutrons from nitrogen and oxygen. We have chosen the neutron energy not only for the practical aspects of the $T(d, n) a$ reaction, but also because at these energies the relatively small contribution of compound elastic scattering permits a more favourable comparison with the complex potential model. It is hoped that our results together with those reported for Li, Be, B, and C (refs. 1-4), will allow a more careful theoretical fitting of the cross sections for light nuclei.

We also present limited cross section data for inelastic scattering to low-lying excited states in nitrogen and oxygen. There have been several previous experiments on neutron scattering at $14 \mathrm{MeV}$ on nitrogen and oxygen. Among the earliest were the cloud chamber measurements on oxygen by Conner ${ }^{8}$ ) and on nitrogen by Smith $\left.{ }^{9}\right)$. More recently Strizhak et al. ${ }^{2}$ ) and Bobyr et al. $\left.{ }^{10}\right)$ reported on elastic and inelastic neutron scattering on nitrogen, respectively. Also, elastic and inelastic scattering data for protons from nitrogen and oxygen at the roughly equivalent energy of 14 to $15 \mathrm{MeV}$ are available 11,12 ). We compare our results with the previously reported neutron and proton differential scattering cross sections.

\section{Experimental procedure}

Figure 1 is a schematic drawing of the experimental setup. The swept and bunched $0.5-\mathrm{MeV}$ deuteron beam of the Cockcroft-Walton accelerator 
strikes a tritium-loaded target and produces short bursts ( $3 \mathrm{nsec}$ ) of 14MeV neutrons.

The annular ring geometry of the scatterer is similar to that used in previous experiments 1,3 ). As scatterers, liquid nitrogen and liquid oxygen at their boiling point near normal atmospheric pressure were used. To contain the liquids a stainless steel ring-shaped container was employed with a mean diameter of $28 \mathrm{~cm}$ and a square cross section of $3.8 \times 3.8 \mathrm{~cm}$. The single steel wall (thickness $0.02 \mathrm{~cm}$ ) of the container was surrounded by a 1.5-cm-thick Styrofoam layer for effective thermal insulation. To insure complete filling of the container and to reduce the accumulation of gas bubbles in the scatterer, a continuous flow system for the liquids was used. From a reservoir about $150 \mathrm{~cm}$ below the scatterer, the container was filled by means of a well-insulated steel transfer tube (inner diameter about $0.5 \mathrm{~cm}$ ). A liquid flow regulator, about $150 \mathrm{~cm}$ above the scatterer, adjusted the gas pressure in the reservoir to support a liquid column about $300 \mathrm{~cm}$ high. Remote-control filling and draining of the container enabled repeated background checks. The background from the container and flow system was about $20 \%$ of the signal from the liquid scatterers. The angle of scattering, $\theta$, was varied by moving the ring scatterer together with the reservoir and flow regulator with respect to the target. This results in a change of the incident energy with scattering angle. At $\theta=20^{\circ}$, the mean incident neutron energy is $14.7 \mathrm{MeV}$; at $90^{\circ}, 14.1 \mathrm{MeV}$; and at $140^{\circ}, 13.6 \mathrm{MeV}$. For convenience, $14 \mathrm{MeV}$ is quoted as the nominal energy.

The neutron detector consisted of a 5-cm-diameter $\times 4.5-\mathrm{cm}$-thick stilbene scintillator, mounted on an RCA 6810A photomultiplier. The detector bias was $3.5 \mathrm{MeV}$ for neutrons (or I MeV for electrons). The detector efficiency was measured at $14.7 \mathrm{MeV}$ by removing the copper absorber which 
was used during the scattering experiment to shield the detector from the source neutrons. The energy dependence of the detector efficiency was meas ured by scattering $14-\mathrm{MeV}$ neutrons from polyethylene ( $\mathrm{n}-\mathrm{p}$ differential scattering). The neutron detector was placed inside a paraffin-and-lead shield for the purpose of reducing the background from the room as well as from the liquid reservoir and the flow regulator. To reduce the background from air scattering, the distance between the tritium target and front end of the copper absorber was adjusted for the following optimum conditions: $43 \mathrm{~cm}$ for small angle scattering $\left(17-70^{\circ}\right) ; 18 \mathrm{~cm}$ for medium angles $\left(30-120^{\circ}\right)$; and $5.4 \mathrm{~cm}$ for large angle scattering $\left(70-140^{\circ}\right)$

The electronic circuits consisted of the standard Livermore time-offlight electronics with the usual single-channel energy selection and multichannel time display. The system has been described previously 13,14 ). A new proton-electron pulse shape discriminating circuit was used to suppress the gamma ray background. The discriminator, similar in design to that described by Batchelor et al. 15$)$, is based upon the pulse-form differences between proton-and electron-induced scintillation light pulses in stilbene. The time-vs-neutron scintillation energy calibration is shown in fig. 2 ; the resolution of the equipment is illustrated in figs. 3 and 4. All three time-of-flight spectra, taken at a scattering angle of $50^{\circ}$, have been corrected for background.

\section{Experimental results}

The observed differential elastic cross sections for $14-\mathrm{MeV}$ neutrons scattered from nitrogen and oxygen are shown in figs. 5 and 6, respectively. The results, presented in the center-of-mass system, have been corrected for absorption and multiple scattering in the liquid targets. No angular resolution correction has been included, since the angular spread at forward angles $\left(\theta=20^{\circ}\right)$ was only $\pm 2^{\circ}$, while in the region of the broad secondary maximum near $90^{\circ}$ it widened to $\pm 6^{\circ}$. 
Great care was taken to minimize systematic errors. The experiments were performed alternately with and without the scattering liquid in the ring, thus eliminating large uncertainties in the background level which may introduce appreciable errors in the cross section measurements. Frequent calibrations of the neutron counter showed no gradual drifts of the electronic system (overall drift less than $5 \%$ over a period of several days). The relative errors, affecting the shape of the angular distribution but not the magnitude of the cross section, have been estimated to be about $\pm 7 \%$ which is a combination of errors due to counting statistics, accuracy of background subtractions, and uncertainty in the efficiency correction. The absolute errors for the differential elastic scattering cross sections are about $\pm 10 \%$ which include uncertainties in the efficiency determination and in the absorption and multiple-scattering corrections.

Using a reasonable extrapolation for angles less than $17^{\circ}$ (see fig. 7 for oxygen) gives for the integrated elastic cross sections: nitrogen, 0.87 \pm 0.09 barn; oxygen, $0.88 \pm 0.09$ barn. Since the total cross sections $\sigma_{T}(N)$ $=1.59 \pm 0.03$ barns 16$)$ and $\sigma_{\mathrm{T}}(0)=1.61 \pm 0.03$ barns 16,17$)$, this implies $\sigma_{\text {non }}(N)=0.72 \pm 0.10$ barn and $\sigma_{\text {non }}(\mathrm{O})=0.73 \pm 0.10$ barn for the nonelastic cross sections. The former value agrees with the nonelastic measurements by Phillips et al. ${ }^{18}$ ) on nitrogen, $\sigma_{\text {non }}(N)=0.79 \pm 0.05$ barn. The measurements by Flerov and Talyzin ${ }^{19}$ ) give $\sigma_{\text {non }}(N)=0.82 \pm 0.02$ barn and $\sigma_{\text {non }}(O)$ $=0.85 \pm 0.03$ barn. Better agreement with our results cannot be expected since the latter authors have not fully corrected their results for elastic energy degradation. (Some large-angle elastic scattering is included in the nonelastic measurement since the energy of the elastic neutrons is degraded by the center-of-mass motion.) 
Using a least-squares fit of our data for scattering angles from 17 to $40^{\circ}$, the extrapolations of the elastic scattering cross sections to zero scattering angle (see fig. 7 for oxygen) give for nitrogen $\sigma_{\mathrm{el}}\left(0^{\circ}\right)=0.97 \pm 0.085$ barn and for oxygen $\sigma_{\mathrm{el}}\left(0^{\circ}\right)=1.11 \pm 0.10$ barns in the laboratory system. It is interesting to compare these values with the Wick limit 20 ) given by $\sigma_{\text {el }}\left(0^{\circ}\right)$ $=\left(\mathrm{k} \sigma_{\mathrm{T}} / 4 \pi\right)^{2}$ where $\mathrm{k}$ is the wave number of the incident neutron. Using the total cross section $\sigma_{\mathrm{T}}$ given above, the Wick limit yields $1.08 \pm 0.04$ barns for nitrogen, and $1.10 \pm 0.04$ barns for oxygen. The good agreement between the extrapolated values and the Wick limits, previously demonstrated for many elements 1,21$)$, greatly reduces the possibility of systematic errors.

The inelastic scattering angular distributions to the $3.95-\mathrm{MeV}$ level in $\mathrm{N}^{14}$ and to the four lowest excited states near 6 and $7 \mathrm{MeV}$ in $\mathrm{O}^{16}$ are also plotted in figs. 5 and 6, respectively. These inelastic cross sections, measured for an angular range from 32 to $140^{\circ}$, have not been corrected for multiple scattering or absorption because the counting statistics are quite poor (about $25 \%$ ) while the multiple scattering and absorption corrections are estimated to be of the order of $15 \%$.

The inelastic scattering angular distribution to the $3.95-\mathrm{MeV}$ level in $\mathrm{N}^{14}$ exhibits a minimum near $\theta=100^{\circ}$ and appears to be slightly peaked in the forward direction (see fig. 5). The inelastic scattering angular distribution to the $2.31-\mathrm{MeV}$ level in $\mathrm{N}^{14}$ was found to have the same general shape as that to the $3.95-\mathrm{MeV}$ level, but the magnitude of the differential cross section was lower by at least a factor of 3 compared with cross sections to the 3.95- MeV level over the whole angular range measured from 32 to $140^{\circ}$. Our measurements indicate differential cross sections to the $2.31-\mathrm{MeV}$ level in $\mathrm{N}^{14}$ for scattering angles of 60,100 , and $140^{\circ}$ to be about $0.5,0.3$, and 0.4 $\mathrm{mb} / \mathrm{sr}$, respectively, with an estimated uncertainty of $0.2 \mathrm{mb} / \mathrm{sr}$. These 
results, typical of the accuracy achieved for our inelastic scattering data to the $2.31-\mathrm{MeV}$ level, are in strong disagreement with the inelastic scattering cross sections reported by Bobyr et al. ${ }^{10}$ ). These authors, though claiming an energy resolution of $3 \%$ for their neutron spectrometer, report inelastic cross sections of $10 \mathrm{mb} / \mathrm{sr}$ and larger for the angular range under discussion. In fact, their inelastic differential cross sections for scattering angles of $50^{\circ}$ and larger are of about the same magnitude as our measured elastic scattering cross section. Our neutron spectra (see fig. 3) are not compatible with such large inelastic cross sections. The integrated cross section for inelas tic scattering to the $2.3-\mathrm{MeV}$ level is $4 \mathrm{mb}$, accurate within a factor of 2 .

The inelastic-scattering differential cross section to the lowest four excited states in $\mathrm{O}^{16}$ is reported here as a sum over the four levels (see fig. 6). Though the two neutron groups to the 6.05- and 6.13-MeV states are resolved from the two neutron groups to the 6.92- and $7.12-\mathrm{MeV}$ states (see fig. 4), we have not attempted to present quantitative inelastic cross sections to the level doublet near $6 \mathrm{MeV}$ and the doublet near $7 \mathrm{MeV}$ separately, since our results still would not allow us a comparison with theoretical predictions available only for individual levels, e.g., for the $3^{-}$collective state at 6.13 $\mathrm{MeV}$ evaluated by Payne and Snider 22 ). Over the angular range measured from 32 to $140^{\circ}$, both angular distributions to the doublet near $6 \mathrm{MeV}$ and to the doublet near $7 \mathrm{MeV}$ exhibit only a weak angular dependence; the former doublet is slightly peaked in the forward direction $\left(\sigma\left(40^{\circ}\right)=11 \pm 1 \mathrm{mb} / \mathrm{sr}\right.$ and $\left.\sigma\left(125^{\circ}\right)=7 \pm 1 \mathrm{mb} / \mathrm{sr}\right)$, while the latter is almost symmetric about a broad minimum at $100^{\circ}\left(\sigma\left(40^{\circ}\right)=6 \pm 1 \mathrm{mb} / \mathrm{sr}, \sigma\left(95^{\circ}\right)=3 \pm 1 \mathrm{mb} / \mathrm{sr}, \sigma\left(125^{\circ}\right)=5\right.$ $\pm 1 \mathrm{mb} / \mathrm{sr}$ ). The integrated cross section for inelastic scattering to the level doublet near $6 \mathrm{MeV}$ is of the order of $90 \mathrm{mb}$; to the doublet near $7 \mathrm{MeV}$ it is of the order of $65 \mathrm{mb}$. These cross sections are considered to be accurate to 
within $40 \%$ where the main errors are due to statistics, difficulties in separating the two doublets from each other, and uncertainties in the extrapolation outside the angular range measured from 32 to $140^{\circ}$.

\section{Discussion}

As seen in figs. 5 and 6 , the angular distributions of the neutrons elas tically scattered from nitrogen and oxygen exhibit a pronounced asymmetry. The strong peaking in the forward direction for both elements was previously observed in the cloud chamber studies and interpreted as diffraction scattering 8,9 ). Our differential elastic scattering cross sections on nitrogen agree with the recently reported results by Strizhak et al. ${ }^{2}$ ) for small angle scattering, but are higher by a factor of about 1.5 for all scattering angles larger than $70^{\circ}$. Though Strizhak et al..$\left.^{2}\right)$ have performed their experiment under experimental conditions similar to ours, they have not corrected for multiple scattering which can account for at least part of the discrepancy between the results.

The solid curves in figs. 5 and 6 are the theoretical results of Bjorklund and Fernbach ${ }^{5}$ ) for $14-\mathrm{MeV}$-neutron elastic scattering from nuclei with mass numbers $\underline{A}=14$ and 16, respectively. They correspond to an optical potential with a spin-orbit coupling term using a single set of parameters describing the model to give the best overall fit to the available experimental data. It is to be expected that the heavier elements are described best (for elements heavier than aluminium, see ref.6) and that the theoretical curves become progressively less reliable as we consider lighter elements. Definite deviations of the measured cross sections for Li, Be, B, and C from the BjorklundFernbach curves have been reported previously $\left.{ }^{1-4}\right)$, in particular for scattering angles larger than $70^{\circ}$. Our results on $\mathrm{N}$ and $\mathrm{O}$ show a similar deviation for scattering angles beyond the first minimum in the differential elastic scattering cross sections. 
Presently attempts are being made to calculate a best single set of parameters to produce an optimum systematic overall fit to the available experimental data for light elements $(Z=3$ to 8$)$. Though it is possible to handtailor curves to each set of experimental data and achieve better theoretical results, we hope to arrive at a systematic compilation of the parameters describing the optical model which will allow, by interpolation, the determination of a usable differential cross section for elastic scattering of $14-\mathrm{MeV}$ neutrons from any element, even the light elements below $Z=13$.

The dashed curves in figs. 5 and 6 represent the elastic and inelastic scattering cross sections for protons from nitrogen and oxygen at the approximately equivalent energy of 14 to $15 \mathrm{MeV}$. The (p,p) and (p,p') data have been reported for nitrogen by Oda et al. ${ }^{11}$ ), for oxygen by Kobayashi ${ }^{12}$ ). To allow a comparison with our inelastic data for oxygen, the ( $\left.p, p^{\prime}\right)$ cross sections have been combined for the lowest four excited states in oxygen (see fig. 6). The good agreement existing between proton and neutron inelastic scattering cross sections can be seen from figs. 5 and 6 . Also the inelastic cross sections to the $2.31-\mathrm{MeV}$ state in $\mathrm{N}^{14}$ and to the level doublet near $6 \mathrm{MeV}$ in $\mathrm{O}^{16}$ and the doublet near $7 \mathrm{MeV}$ in $\mathrm{O}^{16}$, all discussed in section 3, are,within experimental accuracy, the same for proton and neutron scattering. Considering the strong energy dependence of the elastic scattering cross section (for oxygen, see fig. 6), we also find close agreement between proton and neutron data for the elastic process.

Though recently discrepancies between proton and neutron scattering data have been reported, e.g., for small angle inelastic scattering 23,24 ) to the $7.65-\mathrm{MeV}$ level in $\mathrm{C}^{12}$, we find general agreement between the proton and neutron elastic and inelastic scattering cross sections for $\mathrm{N}^{14}$ and $\mathrm{O}^{16}$ in the angular range investigated. Our experimental results fully support theoretical 
predictions using charge symmetry. The proton and neutron distributions are expected to be only slightly different at $14 \mathrm{MeV}$, since Coulomb effects are relatively unimportant at this energy. Our results, however, strongly disagree with the nitrogen inelastic cross sections measured by Bobyr et al. ${ }^{10}$ ), where large discrepancies between neutron and proton data appear to occur over the whole angular range.

This work was done under the auspices of the U. S. Atomic Energy Commission. A preliminary report has been presented at the Washington meeting of the American Physical Society (April, 1963). 


\section{References}

1) M. P. Nakada, J. D. Anderson, C. C. Gardner and C. Wong, Phys . Rev. 110 (1958) 1436

2) V. I. Strizhak, V. V. Bobyr and L. Ya.Grona, Zh. Eksperim. i Teor. Fiz. 41 (1961) 313 - Soviet Phys. JETP 14 (1962) 225

3) C. Wong, J. D. Anderson and J. W. McClure, Nucl. Phys. 33 (1962) 680

4) K. Tesch, Nuc1. Phys. 37 (1962) 412

5) F. Bjorklund and S. Fernbach, University of California Radiation Laboratory Report UCRL-4926-T (Rev.) (1957)

6) F. Bjorklund and S. Fernbach, Phys. Rev. 109 (1958) 1295; and Proceedings of the International Conference on the Nuclear Optical Model, Florida State University Studies No. 32 (Florida State University, Tallahassee, 1959)

7) J. S. Nodvik, C. B. Duke and M. A. Melkanoff, Phys. Rev. 125 (1962) 975; and more recently for oxygen, see C. B. Duke, Phys. Rev. 129 (1963) 681

8) J. P. Conner, Phys. Rev。 89 (1953) 712

9) J. R. Smith, Phys. Rev。 95 (1954) 730

10) V. V. Bobyr, L. Ya. Grona and V. I. Strizhak, Zh. Eksperim.i Teor. Fiz. 41 (1961) 24 - Soviet Phys. JETP 14 (1962) 18

11) Y. Oda, M. Takeda, N. Takano, T. Yamazaki, C. Hu, K. Kikuchi, S。 Kobayashi, K. Matsuda and Y. Nakahara, J. Phys. Soc. Japan 15 (1960) 760

12) S. Kobayashi, J. Phys. Soc. Japan 15 (1960) 1164

13) J. D. Anderson, C. C. Gardner, M. P. Nakada and C. Wong, Phys. Rev. $110(1958) 160$ 


\section{References (Continued)}

14) J. D. Anderson and C. Wong, Nucl. Instr. Methods 15 (1962) 178

15) R. Batchelor, W. B. Gilboy, A. D. Purnell and J. H. Towle, Nucl. Instr. Methods $\underline{8}$ (1960) 146

16) J. H. Coon, E. R. Graves and H. H. Barschall, Phys. Rev. 88 (1952) 562

17) H. L. Poss, E. D. Salant, G. A. Snow and L. C. L. Yuan, Phys. Rev. $\underline{87}(1952) 11$

18) D. D. Phillips, R. W. Davis and E. R. Graves, Phys. Rev. 88 (1952) 600

19) N. N. Flerov and V. M. Talyzin, Atomnaya Energiya 1, No. 4 (1956) 155 - Soviet J.At. Energy 1 (1956) 617

20) A. C. Wick, Atti Reale Accad. Italia, Mem. Classe Sci. Fis. Mat. Nat. 13 (1943) 1203

21) J. H. Coon, R. W. Davis, H. E. Felthauser and D. B. Nicodemus, Phys. Rev. 111 (1958) 250

22) A. L. Payne and D. R. Snider, Nuc1. Phys. 33 (1962) 626

23) J. Rethmeier, C. C. Jonker, M. Rodenburg, J. Hovenier and D. Van der Meulen, Nucl. Phys. 38 (1962) 322

24) R. Bouchez, J。Duclos and P. Perrin, Universite de Grenoble Note Scientifique, $\operatorname{PhN} 62 / 85$ (1962) 


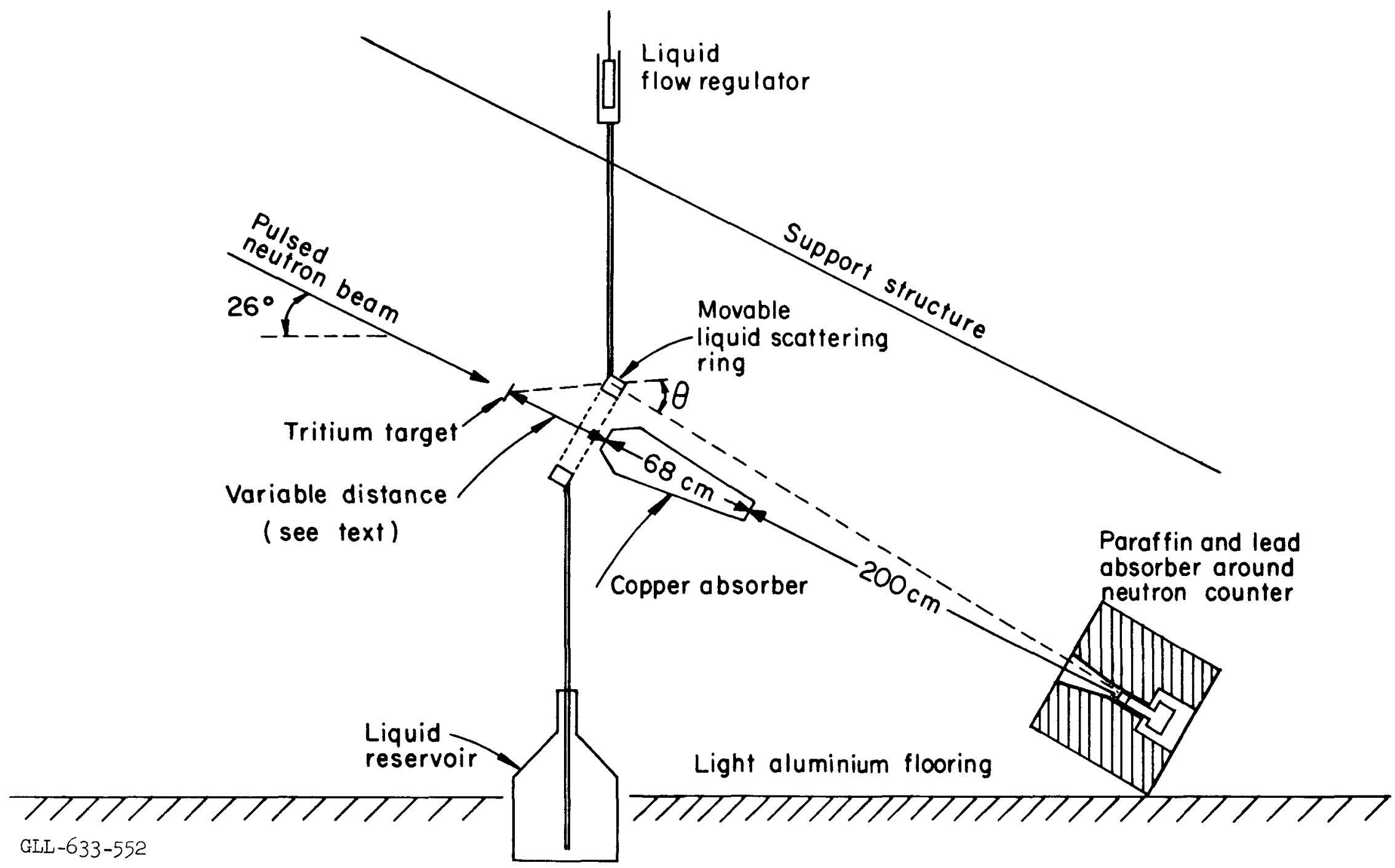

Fig. 1. Schematic diagram of the experimental geometry. The angle of scattering is noted by $\theta$. 


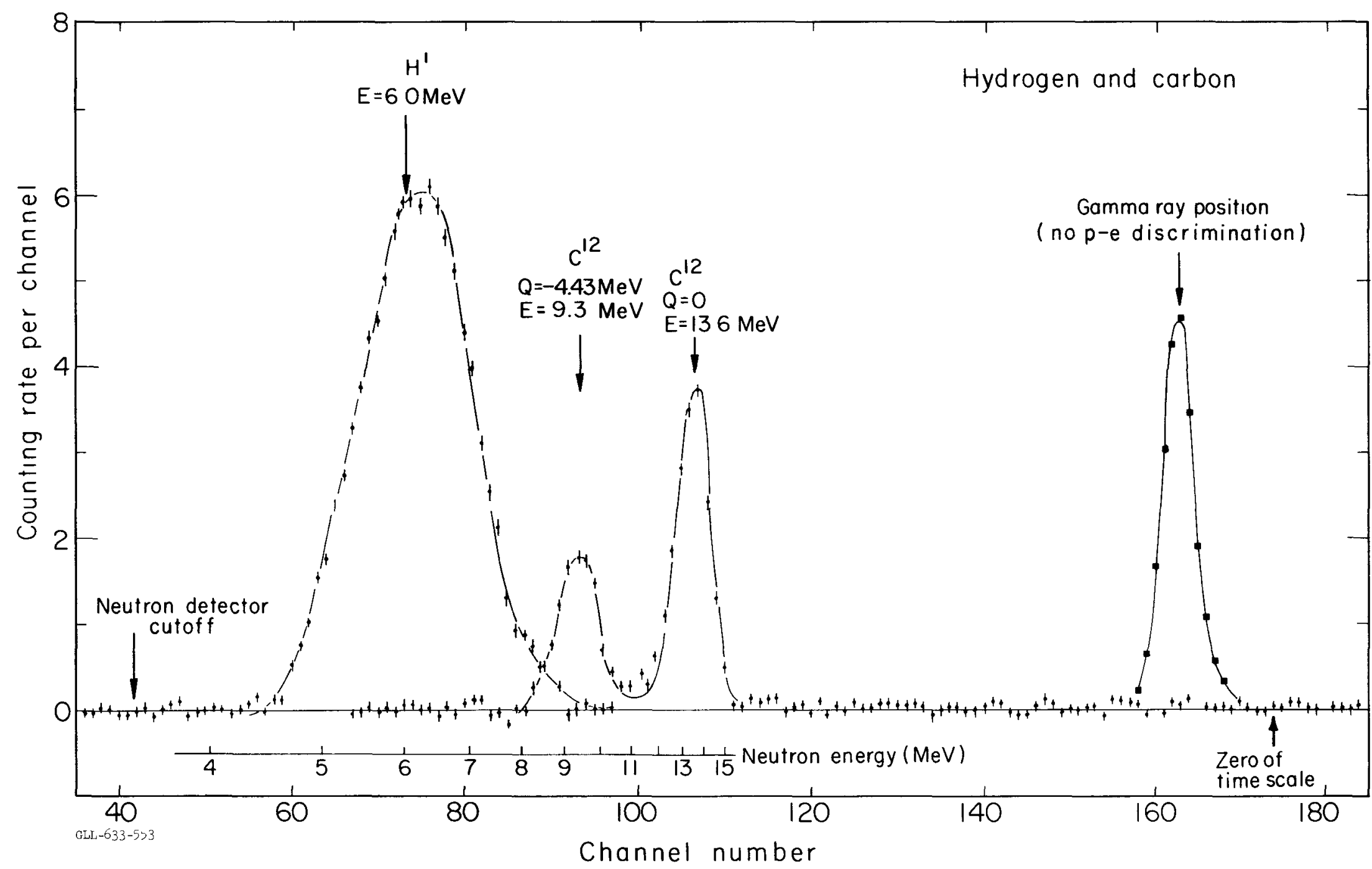

Fig. 2. Time-of-flight spectrum from carbon and hydrogen at a scattering angle of $50^{\circ}$ where the incident neutron energy is $14.5 \mathrm{MeV}$. The time scale is $0.77 \mathrm{nsec}$ per channel, and increasing flight time is toward the left. The neutron energy scale along the abscissa is calculated from the time calibration, flight path, and location of the gamma ray from the scatterer. The energies of the neutron groups in the figure are calculated from kinematics. Note that it is necessary to transform the time to an energy scale to accurately locate the hydrogen scattering peak. 


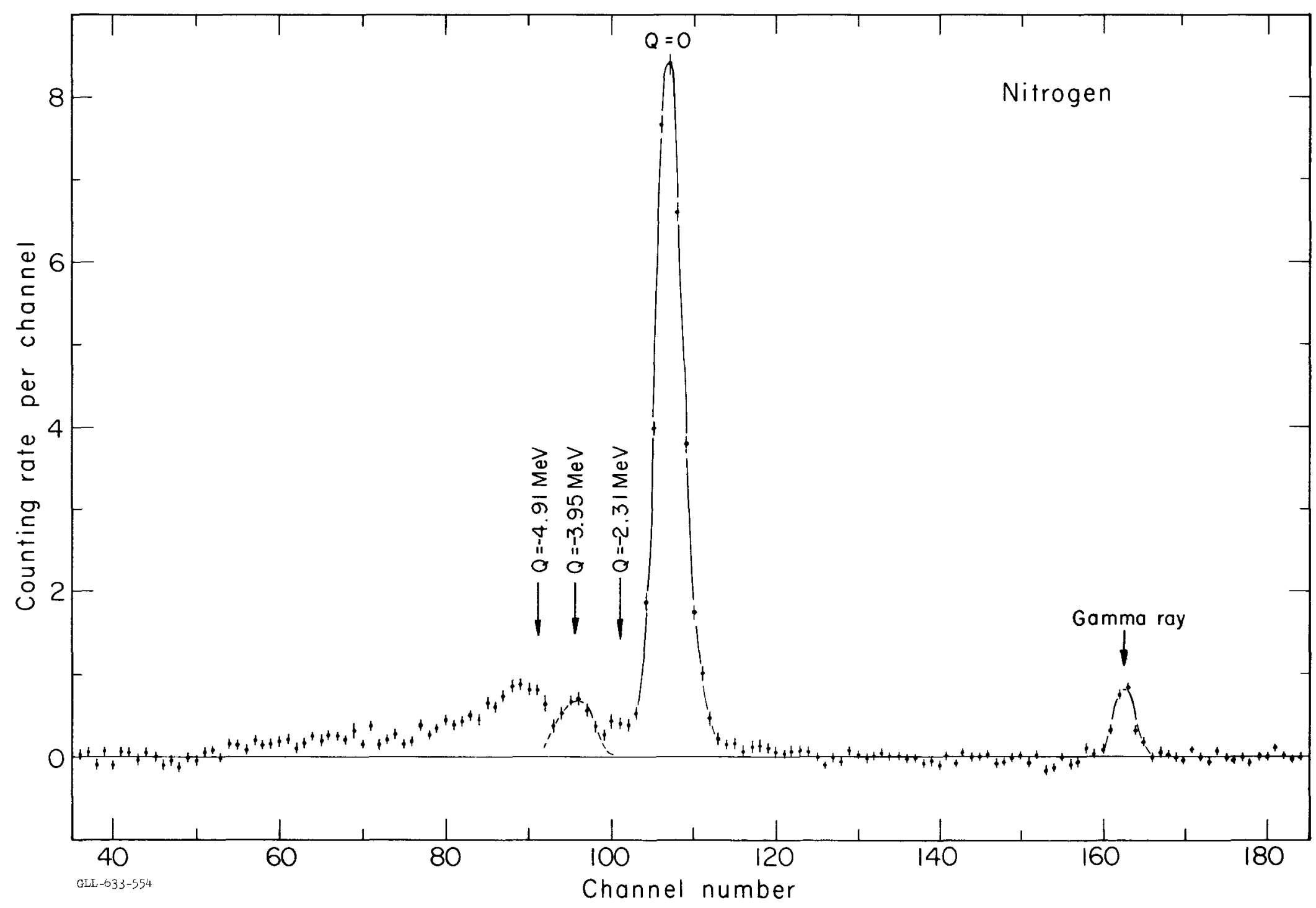

Fig. 3. Time-of-flight spectrum from nitrogen at a scattering angle of $50^{\circ}$. The time and energy scales are the same as in fig. 2. The elastic neutron group is noted by $Q=0$. The inelastic neutron groups from the first two excited states at 2.31 and $3.95 \mathrm{MeV}$ are indicated by their respective $Q$ values. 


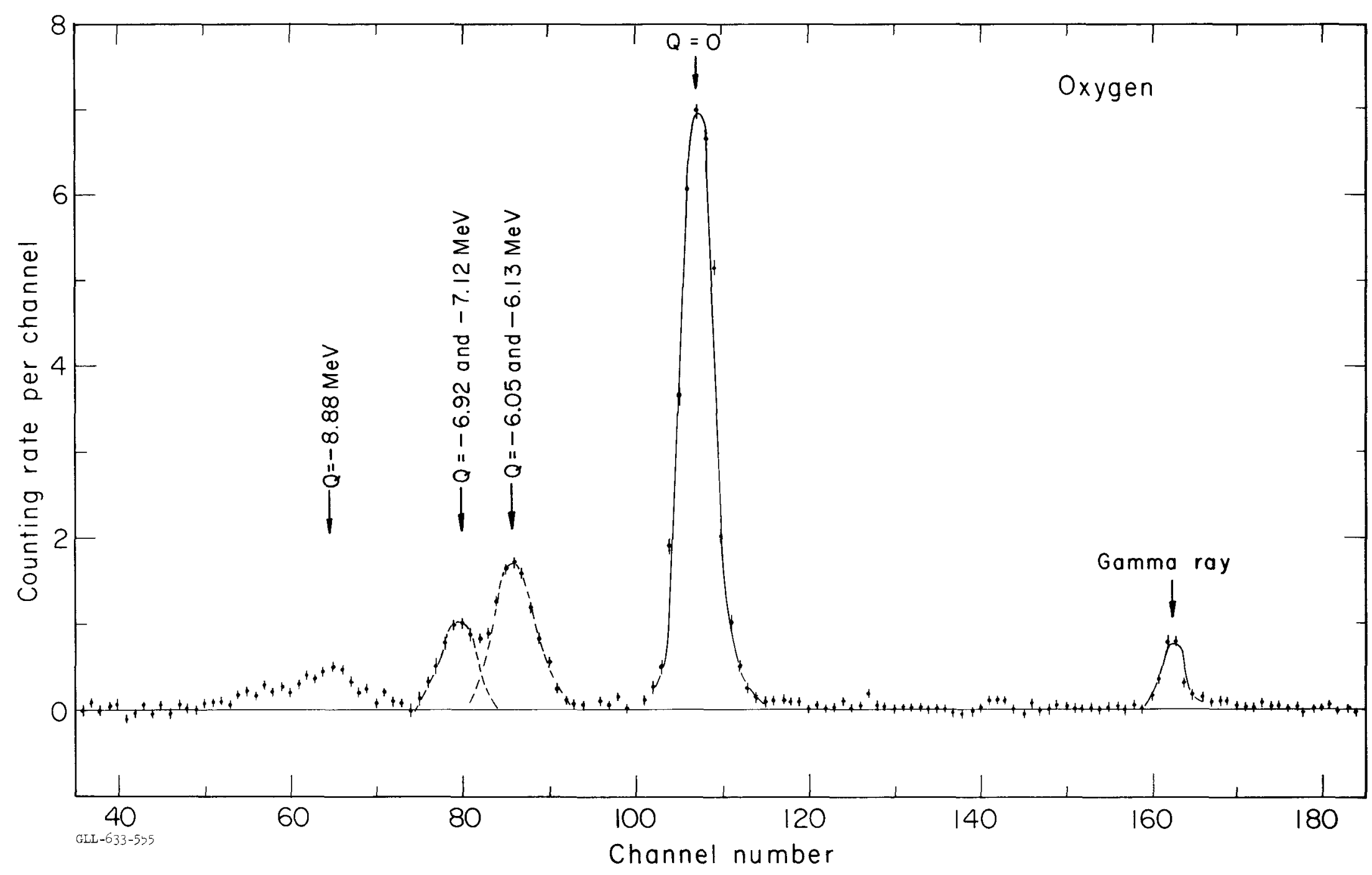

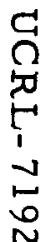

Fig. 4. Time-of-flight spectrum from oxygen at a scattering angle of $50^{\circ}$. The time and energy scales are the same as in fig. 2 , notation same as fig. 3. 


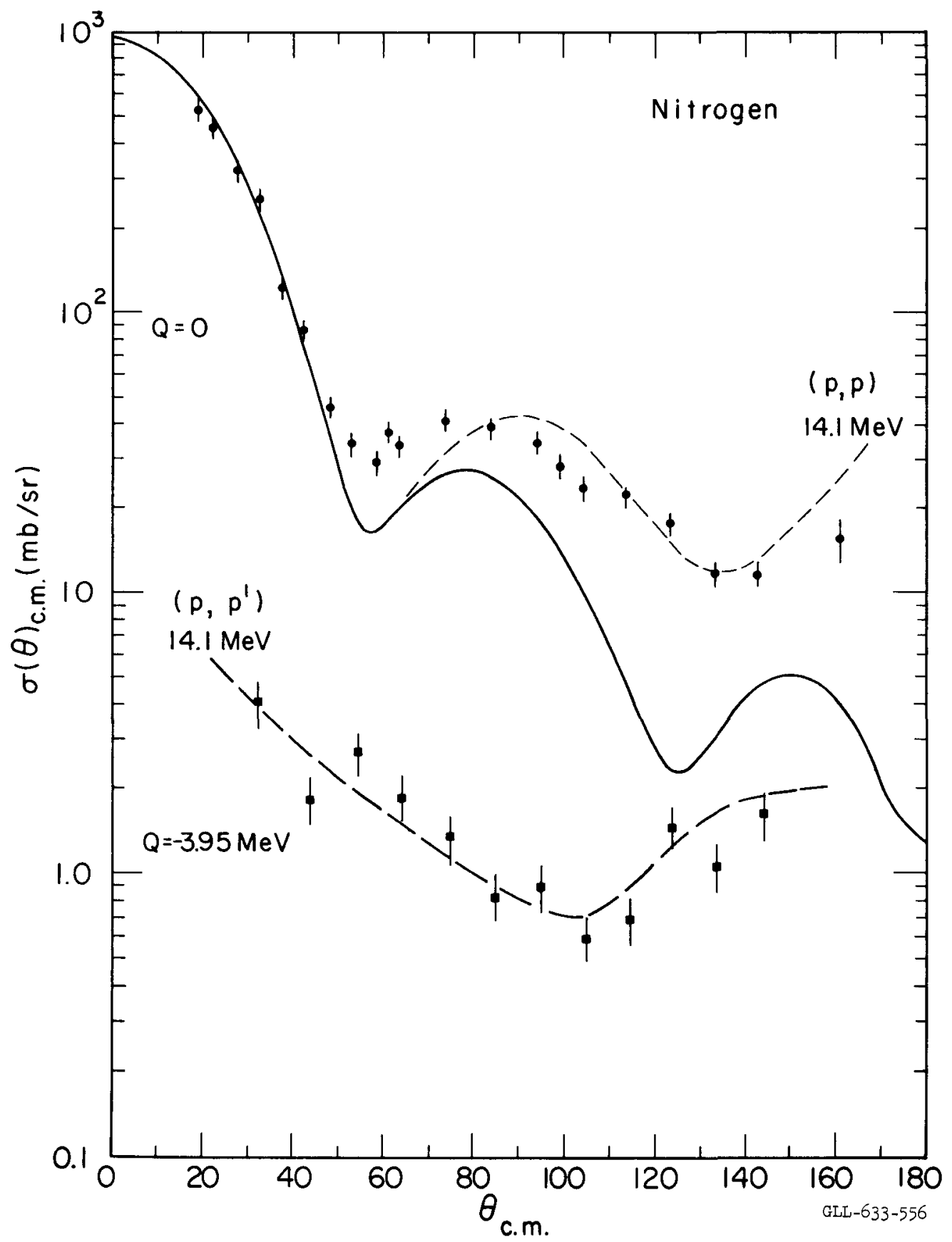

Fig. 5. Elastic and inelastic scattering of $14-\mathrm{MeV}$ neutrons from $\mathrm{N}^{14}$. The circles are the elastic differential cross sections, the squares indicate the inelastic cross sections to the $3.95 \mathrm{MeV}$ level. The solid curve gives the theoretical elastic differential cross sections calculated by Bjorklund and Fernbach (ref. ${ }^{5}$ )). The dashed curves represent the corresponding charged particle data for an incident proton energy of $14.1 \mathrm{MeV}$ (ref. 11 )). 


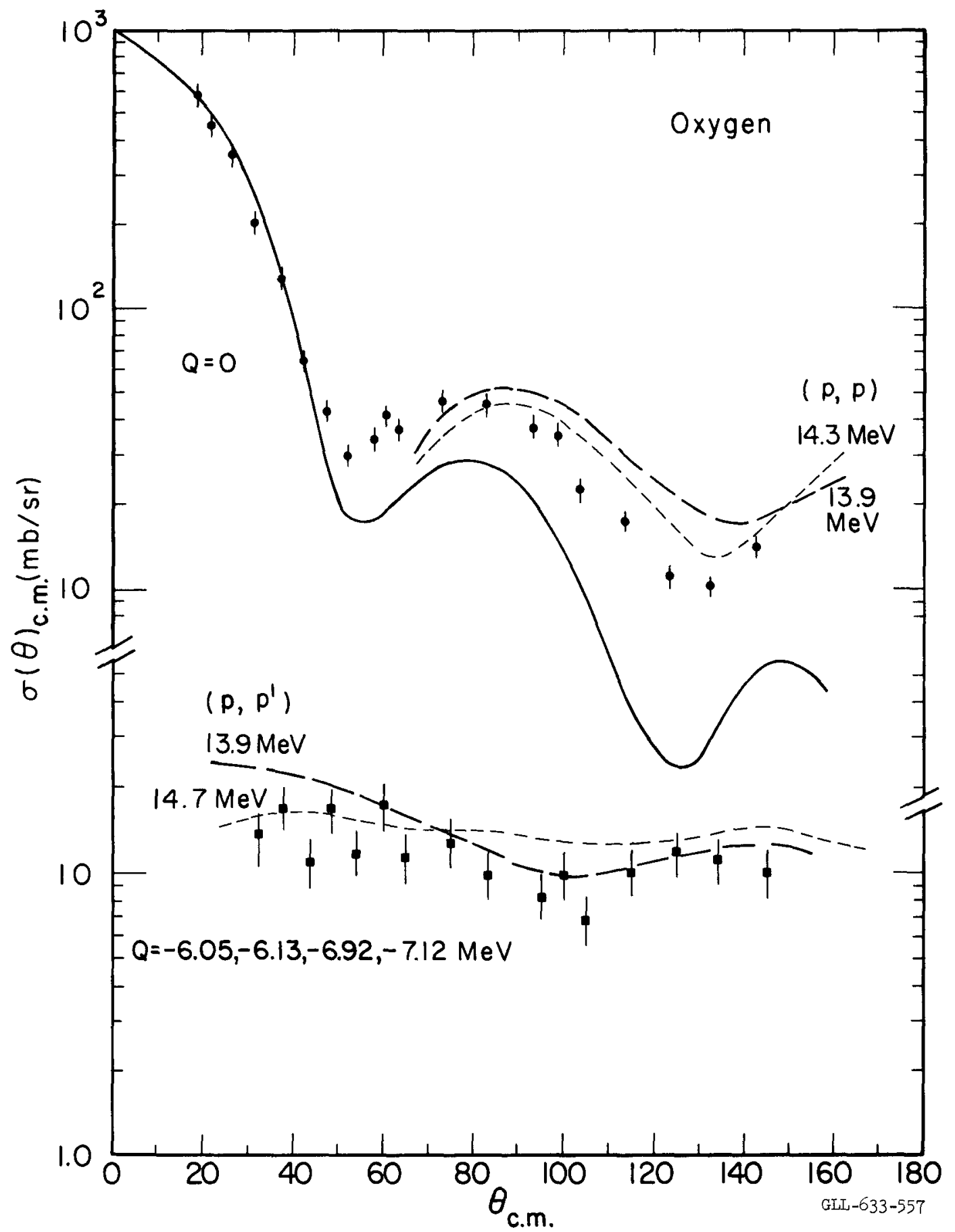

Fig. 6. Elastic and inelastic scattering of $14-\mathrm{MeV}$ neutrons from $\mathrm{O}^{16}$. The circles are the elastic differential cross sections, the squares indicate the inelastic cross sections to the lowest four states at an excitation from 6.05 to $7.12 \mathrm{MeV}$. The solid curve gives the theoretical elastic differential cross sections calculated by Bjorklund and Fernbach (ref. ${ }^{5}$ )). The dashed curves represent the corresponding charged particle data for incident proton energies near $14 \mathrm{MeV}$ (ref. 12 )). 


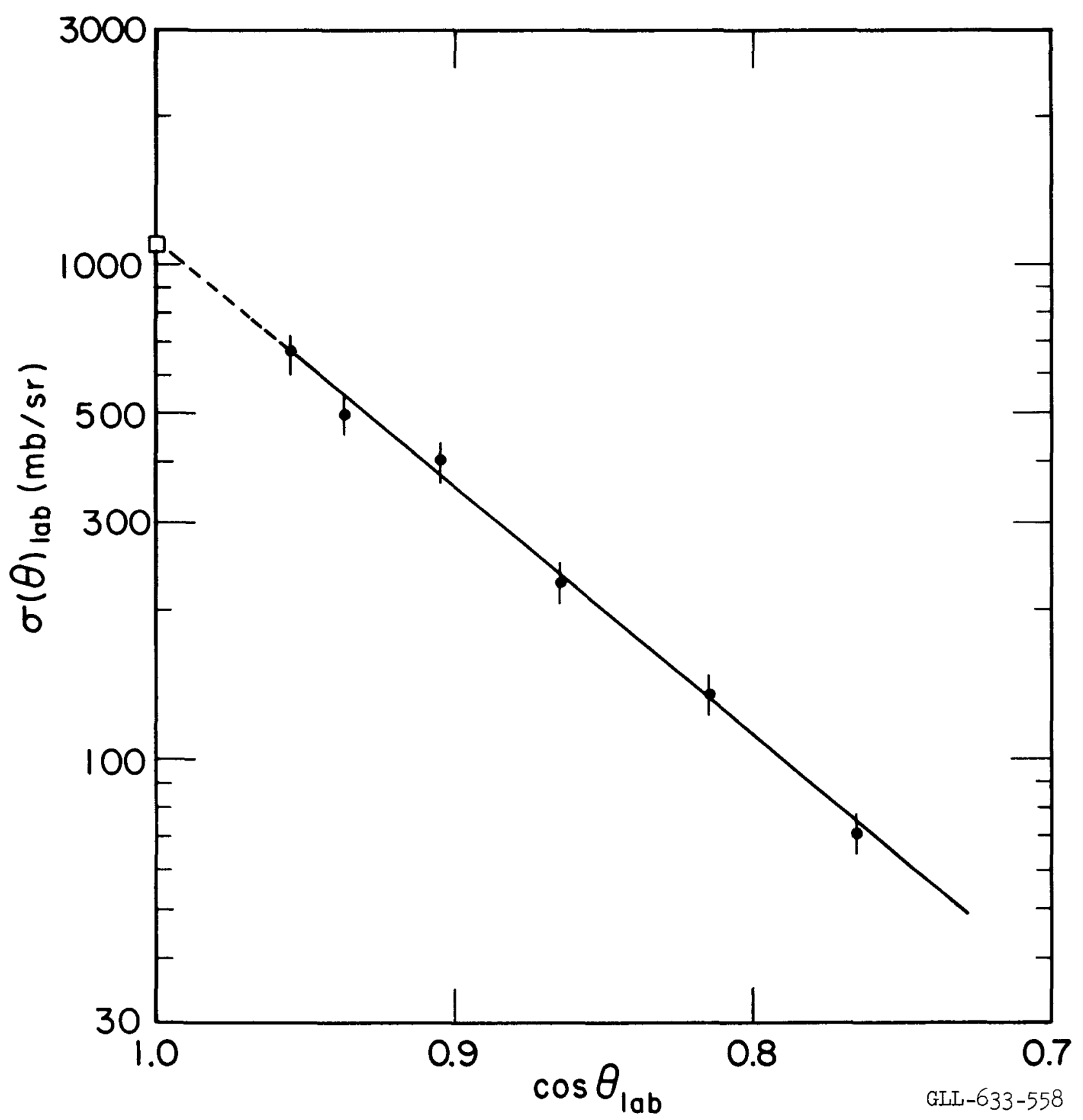

Fig. 7. A semilog plot of the elastic scattering differential cross section $\sigma(\theta)$ versus $\cos \theta$ for oxygen. The dashed portion of the curve represents an extrapolation to angles less than $17^{\circ}$. The square in the ordinate represents Wick's limit, a theoretical lower limit to the differential elastic scattering cross section at $0^{\circ}$ (see text). 
This report was prepared as an account of Government sponsored work. Nelther the United States, nor the Commission, nor any person acting on behalf of the Commission:

A. Makes any warranty or representation, expressed or implied, with respect to the accuracy, completeness, or usefulness of the information contained in this report, or that the use of any information, apparatus, method, or process disclosed in this report may not infringe privately owned rights; or

B. Assumes any liabilities with respect to the use of, or for damages resulting from the use of any information, apparatus, method or process disclosed in this report.

As used in the above, "person acting on behalf of the Commission " includes any employee or contractor of the commission, or employee of such contractor, to the extent that such employee or contractor of the Commission, or employee of such contractor prepares, disseminates, or provides access to, any information pursuant to his employment or contract with the Commission, or his employment with such contractor. 